\title{
The Neuropathological Triangle in Elderly
}

\author{
L Isaac Corona* \\ Geriatric service of the Hospital General Regional 72, National Institute of Social Security, Mexico
}

*Corresponding author: L Isaac Corona, Geriatric service of the Hospital General Regional 72, National Institute of Social Security, Mexico

\begin{tabular}{|c|c|}
\hline ARTICLE INFO & ABSTRACT \\
\hline Received: 蔧 January 27, 2020 & Keywords: Hypertension; Heart Failure; Epilepsy; Dementia; Brain Tumor; Neuroimage; \\
\hline Published: 慧 February 07, 2020 & Atherosclerosis \\
\hline
\end{tabular}

Citation: L Isaac Corona. The

Abbreviation: CVD: Cerebrovascular Disease; AD: Alzheimer's Disease; AED: Neuropathological Triangle in Elderly. Biomed J Sci \& Tech Res 25(3)-2020. BJSTR. MS.ID.004199.

\section{Introduction}

Besides the chronical illness like diabetes, arterial hypertension and heart failure, the geriatric patients have a lot of lacks in other levels. The neurological system is probably the most affected through the years. There are a lot of syndromes and illness because the changes produced by the aging, but in frequencies, it could be summarized in three: vascular pathology, epilepsy, and cognitive disorders. Sen et al., explained previously the relationship between some of this pathologies [1], and also, they show how these conditions are linked. I explain briefly some considerations in elderly patients with one of three main neurological conditions, especially with epilepsy, in a way that could facilitate the approach in the elderly patient who came to see a doctor. According with the literature, the elderly people with epilepsy are those $>65$ years old of age, and it could be separated in two groups: those who had epilepsy since the childhood or young adults and aged with the disease. The second group are those who were diagnosed with epilepsy in the late life.

Cerebrovascular Disease (CVD) is the most common etiology of epilepsy in the elderly, according to Acharya [2],the range of frequency is $20-50 \%$, followed by dementia, brain tumor and head injury. For this etiologies, the elderly patient with an episode of epilepsy, need to be approach with an accurate medical history, a complete neurological examination, and neuroimage. The prevalence of seizures in people with Alzheimer's Disease (AD) is $1-22 \%$, and apparently the risk increases in the late stages, induced by the accumulation of the amyloid-beta that creates aberrant networks causing epileptiform activity [3]. Baker et al., found an accelerated decline in attention, fluency and memory in patients with suspected epileptic seizures [4]. The elderly with advanced cognitive disorder could have an episode of epilepsy with a postictal condition that may be difficult to diagnose, in some cases, the patient could have a transient focal weakness after seizure, but days latter the patient recover the movement in the extremities (Todd's paralysis) [5]. This condition could be considered for the physicians like a transient ischemic attack and prescribe the wrong treatment. Delirium is a common condition in the patients with cognitive disorder, most of the cases could be a sing of acute infection, stroke, or dehydration. If none of this conditions are the factor that triggers the delirium, it must be approached like a possible epileptic manifestation, type impaired awareness non motor onset [6]. Patients with epilepsy have an increased risk to develop cognitive disorders, if the duration of the seizure is prolonged, the risk of brain injuries that can result in cognitive impairment increase [7].

The cognitive decline could be explained not only for the etiology of the epileptic disorder, but the type and frequency, age of onset, the location, and the epileptogenic region [8].The use of Antiepileptic Drugs (AEDs) are associated with cognitive deficits specialy with older AEDs [9]. Recently, Wang et al., showed that patients with epilepsy had a lower cognitive function in vocabulary, 
attention, memory, executive function, and psychomotor speed, affected by educational level, seizure types, seizure frequency, disease duration, depression, age, and AED's [10]. All the elderly patient with epilepsy should have a cognitive assessment, in order to identify some of the deficits in memory or executive function that could lead to forget to take the medication or affect directly to their day living activities.

Finally, there are evidence that patients with epilepsy have an increased risk of stroke not only for the comorbidities but also with the use of AED's for the increase in atherosclerosis level in common carotid artery [11]. Chang et al. showed a 3-fold higher incidence for stroke in the epilepsy cohort than in the comparison cohort and also 6.3-fold higher for the patients aged 60 years than for those aged 20-39 years, with higher risk for ischemic stroke than hemorrhagic stroke. This was explained by the harmful effects of recurrent epilepsy and vascular risk factors contribute to the overproduction of reactive oxygen species and the oxidation of low-density lipoproteins into atherogenic particles, leading to atherosclerosis [12]. The elderly patient needs to be assess for atrial fibrillation and CVD to reduce this risk. As we can see, the epilepsy could be a challenge to diagnose or treat as age progresses and the manifestation of the disease becomes more atypical. If the elderly patient with a neurological condition is not well approached, the consequences could be harmful and lead the patient to polypharmacy, frequent hospitalizations and death.

\section{References}

1. Sen A, Capelli V, Husain M (2018) Cognition and dementia in older patients with epilepsy. Brain 141(6): 1592-1608.

\section{ISSN: 2574-1241}

DOI: 10.26717/BJSTR.2020.25.004199

L Isaac Corona. Biomed J Sci \& Tech Res

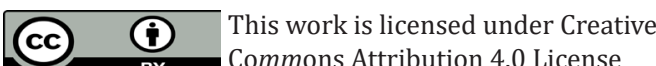

Submission Link: https://biomedres.us/submit-manuscript.php
2. Jayant N Acharya, Vinita J Acharya (2014) Epilepsy in the elderly: Special considerations and challenges. Annals of Indian Academy of Neurology 17(Suppl 1): S18-S26.

3. Nicastro N, Assal F, Seeck M (2016) From here to epilepsy: the risk of seizure in patients with Alzheimer's disease. Epileptic Disorders 18(1): $1-12$.

4. John Baker, Tina Libretto, William Henley, Adam Zeman A (2019) Longitudinal Study of Epileptic Seizures in Alzheimer's Disease. Frontiers in Neurology 10: 1266.

5. Theodore WH (2010) The postictal state: Effects of age and underlying brain dysfunction. Epilepsy Behavior 19(2): 118-120.

6. Pack A M (2019) Epilepsy Overview and Revised Classification of Seizures and Epilepsies. Continuum Lifelong Learning in Neurology 25(2): 306-321.

7. Homes G L (2015) Cognitive impairment in Epilepsy: The Role of Network Abnormalities. Epileptic Disorders 17(2): 101-116.

8. Carreño M, Donaire A, Sánchez Carpintero R (2008) Cognitive disorders associated with epilepsy: diagnosis and treatment. Neurologist 14(6 Suppl 1): S26-S34

9. Sung Pa P, Soon Hak K (2008) Cognitive Effects of Antiepileptic Drugs. J Clin Neurol 4(3): 99-106.

10. Wang L, Chen S, Liu C, Lin W, Huang H (2020) Factors for cognitive impairment in adult epileptic patients. Brain and Behavior 10: e01475.

11. Tan TY, Lu CH, Chuang HY, Lin TK, Liou CW et al. (2009) Longterm antiepileptic drug therapy contributes to the acceleration of atherosclerosis. Epilepsia 50(6): 1579-1586.

12. Chang CS, Liao CH, Lin CC, Lane HY, Sung FC et al. (2014) Patients with epilepsy are at an increased risk of subsequent stroke: A populationbased cohort study. Seizure 23(5): 377-381.

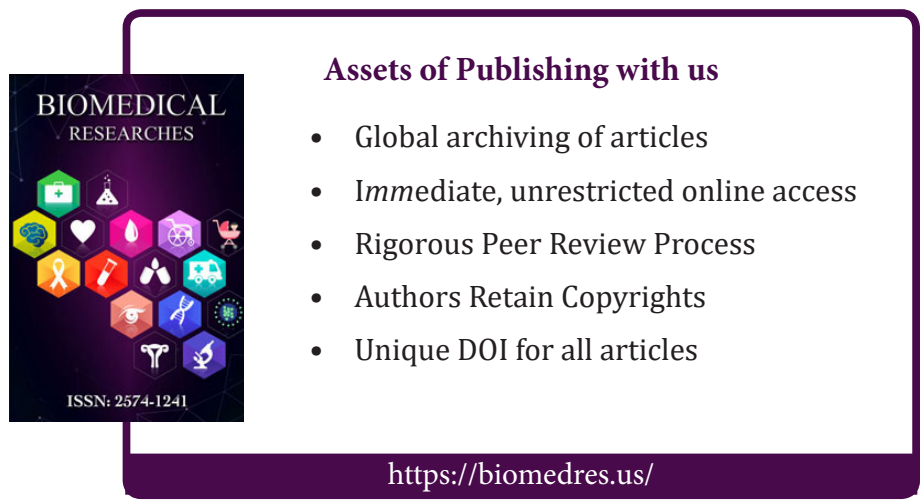

